\title{
Novel compound heterozygous $P K H D 1$ mutations cause autosomal recessive polycystic kidney disease in a Han Chinese family
}

\author{
JIN WANG $^{1 *}$, DANDAN QI $^{1 *}$, JIALIANG YANG $^{1}$, DINGDING ZHANG $^{1}$, \\ QINGWEI WANG ${ }^{1}$, XUEMING JU ${ }^{2}$ and XIANG ZHONG ${ }^{3}$ \\ Departments of ${ }^{1}$ Laboratory Medicine, ${ }^{2}$ Ultrasound and ${ }^{3}$ Nephrology, \\ Sichuan Academy of Medical Sciences and Sichuan Provincial People's Hospital, School of Medicine, \\ University of Electronic Science and Technology of China, Chengdu, Sichuan 610072, P.R. China
}

Received February 8, 2019; Accepted July 8, 2019

DOI: $10.3892 / \mathrm{mmr} .2019 .10738$

\begin{abstract}
Autosomal recessive polycystic kidney disease (ARPKD) is a hereditary fibrocystic disease that primarily involves the kidneys and hepatobiliary tract. The polycystic kidney and hepatic disease 1 (PKHDI) gene is the only gene implicated in ARPKD. The present study aimed to identify PKHD1 mutations causing ARPKD in a Chinese family. A couple that underwent prenatal genetic diagnosis for ARPKD and their families were recruited for the present study. Genomic DNA was collected from the amniotic fluid of the fetus (proband) and from peripheral blood of all other available family members. Targeted exome sequencing was performed on the couple and the proband, followed by direct Sanger sequencing on other family members and normal controls to confirm candidate pathogenic variants. Two novel compound heterozygous mutations in the PKHDI gene were identified as causative in the proband, including maternally inherited c.2876C $>$ T (p.Ser959Phe) and paternally inherited c.5772C >A (p.Phe1924Leu). Each mutation was found to
\end{abstract}

Correspondence to: Dr Xiang Zhong, Department of Nephrology, Sichuan Academy of Medical Sciences and Sichuan Provincial People's Hospital, School of Medicine, University of Electronic Science and Technology of China, 32 West Section 2, Ring 1 Road, Chengdu, Sichuan 610072, P.R. China

E-mail: zxiang626@126.com

Dr Xueming Ju, Department of Ultrasound, Sichuan Academy of Medical Sciences and Sichuan Provincial People's Hospital, School of Medicine, University of Electronic Science and Technology of China, 32, West Section 2, Ring 1 Road, Chengdu, Sichuan 610072, P.R. China

E-mail: juxueming@126.com

*Contributed equally

Abbreviations: ARPKD, autosomal recessive polycystic kidney disease; $P K H D 1$, polycystic kidney and hepatic disease 1; NGS, next-generation sequencing

Key words: ARPKD, PKHD1, compound heterozygous mutation co-segregate with the ARPKD phenotype in the family. Other family members either carried one of the two mutations or lacked both mutations, while the mutations were not found in 576 ethnically matched normal controls. Therefore, two novel compound heterozygous $P K H D 1$ mutations were implicated in causing ARPKD in a Han Chinese family. The results expand the mutation spectrum of $P K H D 1$ that leads to ARPKD, which may improve genetic counseling and prenatal diagnosis for families with ARPKD.

\section{Introduction}

Autosomal recessive polycystic kidney disease (ARPKD) is a rare hereditary form of polycystic kidney disease that typically presents in the perinatal period and occurs in 1 in 20,000-40,000 live births worldwide (1). The polycystic kidney and hepatic disease 1 (PKHDI) gene is the only gene that has been identified to cause ARPKD. The longest PKHDI transcript encodes a 4,074 amino acid protein called fibrocystin or polyductin (1-3). This protein is present in fetal and adult kidney cells, and is also expressed in the liver and pancreas (3), albeit at lower levels. Similar to other cystoproteins, polyductin is localized to the basal body and primary cilia of renal and bile duct epithelial cells $(1,4)$. Defects in polyductin may disrupt the normal function of renal cilia. ARPKD is caused by mutations in the PKHDl gene, located at the chromosome 6p12.2 $(1,2)$. The largest $P K H D 1$ gene mutation database contains 748 different mutations (http://www.humgen.rwth-aachen.de). The most common mutation is a missense mutation in exon 3 (c.107C > T), accounting for $20 \%$ of all cases (http://www. humgen.rwth-aachen.de). Most patients with ARPKD present compound heterozygosity, carrying two mutations in two different alleles. Due to the diversity of $P K H D 1$ gene mutations, it is difficult to associate the genotype with the phenotype of ARPKD. At present, it is not understood how genetic alterations in $P K H D I$ lead to the formation of numerous cysts characteristic of polycystic kidney disease.

Recently, targeted exome sequencing has been successfully used to identify genes that cause Mendelian disorders (5). Together, DNA capture technology and next-generation sequencing (NGS) analysis allow for the rapid and cost-effective parallel sequencing of specific genes of interest. In previous studies, targeted exome 
sequencing has been used as a tool for the molecular diagnosis of ARPKD $(1,5,6)$. Using this approach, the present study identified two novel compound heterozygous mutations in the $P K H D l$ gene causing ARPKD in a Chinese family.

\section{Materials and methods}

Subjects. A couple (age, 26 and 27) that underwent prenatal genetic diagnosis for ARPKD were recruited from Sichuan Provincial People's Hospital for the present study (Fig. 1). The patients, one male and one female, were recruited in July 2016. All the subjects underwent comprehensive examinations and had no other related diseases. The present study was approved by the Institutional Review Board of Sichuan Provincial People's Hospital. Written informed consent was obtained from all subjects prior to the present study. There was no history of genetic diseases in the other family members recruited for the present study. Renal ultrasonography in the father (II:4) and mother (II:3) returned normal findings.

DNA extraction. Genomic DNA was collected from the amniotic fluid of the fetus (proband, III:1) and from peripheral blood of all other subjects. Genomic DNA was extracted using a QIAamp DNA Blood Midi kit (Qiagen $\mathrm{GmbH}$ ), according to the manufacturer's protocol. DNA samples were stored at $-20^{\circ} \mathrm{C}$ until use. DNA integrity was evaluated using a NanoDrop spectrophotometer (NanoDrop Technologies; Thermo Fisher Scientific, Inc.).

Targeted NGS. DNA samples from the couple and the proband were analyzed using targeted NGS as reported previously (5). A customdesigned gene panel, synthesized using the Agilent SureSelect Target Enrichment technique(Agilent Technologies, Inc.), was used to capture the coding regions of 356 genes, including their exons and exon-intron boundaries (1.285 Mbp in total). The variants detected were annotated and filtered based on public and inhouse databases: i) Variants within intergenic, intronic and untranslated regions, and synonymous mutations were excluded from downstream analysis; ii) variants in dbSNP138 (www.ncbi.nlm.nih.gov/projects/SNP), the 1000 Genomes Project (ftp.1000genomes.ebi.ac.uk/vol1/ftp), and HapMap Project (ftp.ncbi.nlm.nih.gov/hapmap) were excluded. Homozygous and compound heterozygous gene variations with autosomal recessive heredity were regarded as likely causative variations. Validation and parental origin analysis were performed for the identified variations using conventional Sanger sequencing. Causative mutations were verified using direct sequencing in all family members and 576 normal controls. Primers [PKHD1-exon 27-forward (F), 5'-ggggcagagaaggaacatttg-3', and reverse (R), 5'-agaccctcc ccagattacca-3'; PKHD1-exon 36-F, 5'-caatacttatactatcccgcc ca-3' and R, 5'-aagtttccetcctccatccc-3'] were designed based on genomic sequences from the Human Genome database (www.ncbi.nlm.nih.gov/genome) and were synthesized by Invitrogen (Thermo Fisher Scientific, Inc). Direct sequencing was performed by Sanger sequencing according to the ABI Big Dye sequencing protocols (cat. no. 4376484; Applied Biosystems; Thermo Fisher Scientific, Inc.) and processed samples were sequenced using an ABI3130XL genetic analyzer (Applied Biosystems; Thermo Fisher Scientific, Inc.).
Finally, the possible damaging effects of the mutations were predicted using SIFT (http://provean.jcvi.org/index.php) and PolyPhen (http:/genetics.bwh.harvard.edu/pph2). The human PKHD1 protein was aligned with other PKHD1 proteins to examine the conservation of the residues using homologene (www.ncbi.nlm.nih.gov/homologene).

\section{Results}

Clinical data. A three-generation family was recruited from Sichuan Provincial People's Hospital (Fig. 1). Ultrasound examinations identified two affected individuals (III:1 and III:2) among the eight examined family members. The two affected members in this family exhibited similar clinical features and both died before birth. The first pregnancy of the couple (for the proband, III:1) ended with an induced abortion at 26 weeks. Ultrasound examination for the bilateral kidneys of the fetus (III:1) exhibited enlargement of the kidneys, and polycystic alterations were identified by an abdominal B scan. The echo of the renal parenchyma was increased diffusely, the demarcation of the cortex and medulla was not clear, and the volume of amniotic fluid was also decreased (Fig. 2). In the current pregnancy (for III:2), repeated prenatal ultrasound examinations during weeks 22-26 of gestation exhibited enlargement of the kidneys and polycystic alterations; in addition, the parenchymal echo was enhanced and the boundary of the cortex and medulla was not clear (Table I and Fig. S1). The size of the left kidney was $48.3 \times 30.6 \mathrm{~mm}$, the left renal parenchymal thickness was uneven and $\sim 1.7 \mathrm{~mm}$ thinner than normal. The left kidney also revealed renal sinus separation with a diameter of $18 \mathrm{~mm}$ and caliectasis. The right kidney was $35.1 \times 22.5 \mathrm{~mm}$ in size, renal sinus separation was observed with a diameter of $\sim 8.4 \mathrm{~mm}$ with caliectasis. Furthermore, the thickness of the right renal parenchymal was $3.7 \mathrm{~mm}$. Double renal parenchymal echogenicity was obvious. Since ultrasound examinations could not produce conclusive results, the couple was referred for genetic counseling and molecular prenatal diagnosis.

Mutation analysis. The percentage of readable bases and the coverage depth in the targeted region were used to assess the quality and reliability of the targeted NGS data, and to ensure complete sequencing coverage of all coding regions in candidate genes. The coverage depth was $<200 \mathrm{X}$ and $100 \%$ of bases in coding regions were readable. On average, 645 variations within the 356 genes were covered in the samples analyzed. In this family, the disease exhibited a pattern of recessive inheritance. Under the autosomal recessive model, the filtered data were reduced to two compound heterozygous variants, c.2876C $>$ T (p.S959F) and c.5772C >A (p.F1924L) in the PKHDl gene (NM_138694.3), which exhibited complete co-segregation with the disease. The two compound heterozygous variants were further validated using the Sanger sequencing method in other family members and 576 normal controls. Finally, it was revealed that the uncle (II:1), mother (II:3), father (II:4) and grandmother (I:2) were heterozygous carriers (Table II). The two mutations were absent in the unaffected family member (I:1) and the normal controls. Therefore, the two mutations co-segregated with the phenotype in this family. The results demonstrated that the compound heterozygous mutations c.2876C $>$ T (p.S959F) and c.5772C $>\mathrm{A}$ 


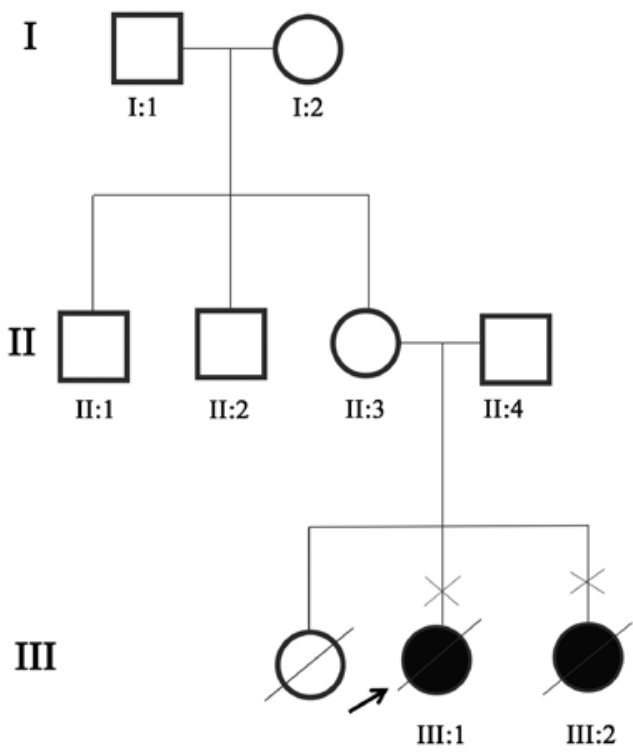

Figure 1. Pedigree of the family with autosomal recessive polycystic kidney disease. Solid symbols indicate affected individuals; open symbols indicate unaffected individuals; squares indicate male individuals; circles indicate female individuals; crosses indicate miscarriages; the arrow indicates the proband and the slash indicates a deceased individual.

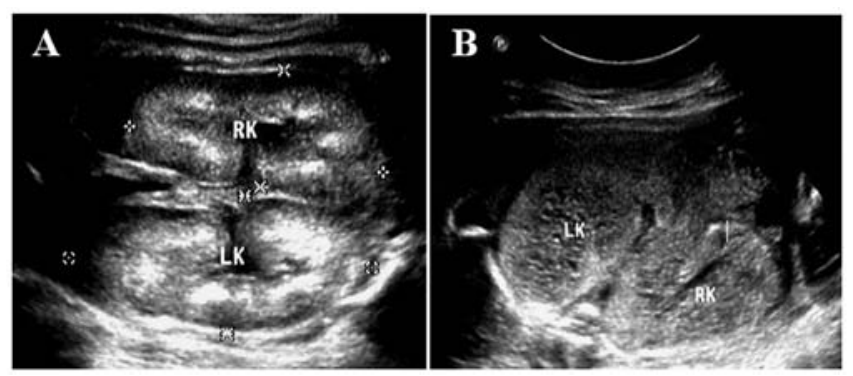

Figure 2. Abdominal ultrasound examinations for the fetus (III:1) at 26 weeks. (A) On the coronal section of the abdomen of the fetus, the size of the two kidneys was increased markedly, the echo of the renal parenchyma was increased diffusely and the volume of amniotic fluid was decreased. (B) A cross-section of the abdomen of the fetus exhibited microcystic alterations in both kidney parenchyma and fibrocystic alterations in the portal vein area of the liver parenchyma. LK, left kidney; RK, right kidney.

(p.F1924L) in the $P K H D 1$ gene are responsible for ARPKD (Fig. 3). Comparative amino acid sequence alignment of other PKHDI proteins across different species revealed that the mutations occurred at highly conserved positions in exons 27 and 36 (data not shown). Furthermore, the two compound heterozygous mutations were predicted to be damaging by the SIFT and PolyPhen tools (Table II). The substituted amino acid was predicted to alter the hydrophobicity of the PKHDI protein. For c.2876C $>$ T (p.S959F) mutation, the hydrophilic serine is changed to a hydrophobic phenylalanine at position 959, while the phenylalanine is changed to leucine at position 1,924 , potentially affecting the structure of the protein, for c.5772C >A (p.F1924L) mutation.

\section{Discussion}

As increased echogenicity and renal enlargement are the main indicators of ARPKD from an ultrasound (1,7); notably, 
Table II. Two novel polycystic kidney and hepatic disease 1 mutations identified in a family with autosomal recessive polycystic kidney disease.

\begin{tabular}{lcccccc}
\hline Position $^{\mathrm{a}}$ & Exon & Nucleotide change & Amino acid change & SIFT score/PolyPhen & Mutant type & Mutation present \\
\hline 51907878 & 27 & c.2876C $>$ T & S959F & $0.03 / 1.0$ (Damaging) & Het & II:4, III: 1 , III:2 \\
51824804 & 36 & c.5772C $>$ A & F1924L & $0.20 / 1.0$ (Damaging) & Het & I:2, II: 1, II: 2, \\
& & & & & & II:3, III: 1, III:2 \\
\hline
\end{tabular}

${ }^{\mathrm{a}}$ Genomic positions are presented according to UCSC hg19 (genome.ucsc.edu). Het, heterozygous.

A Wild-type
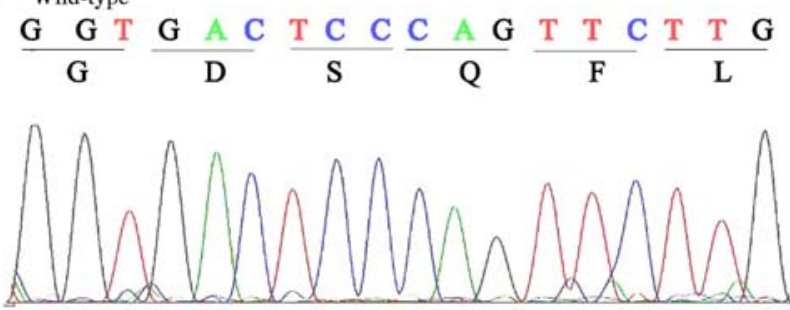

Heterozygous mutant

c. 2876 C $>$ T p.ser 959 phe

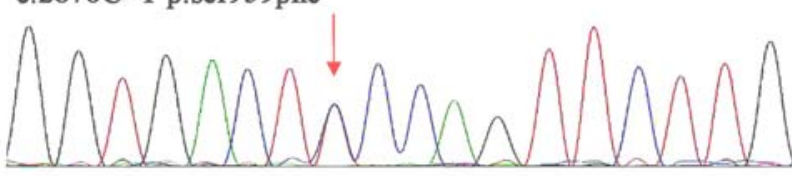

B

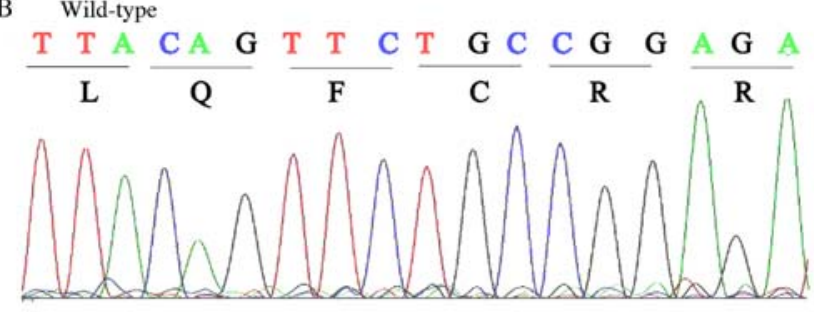

Heterozygous mutant

c.5772C $>$ A p.phe1924leu

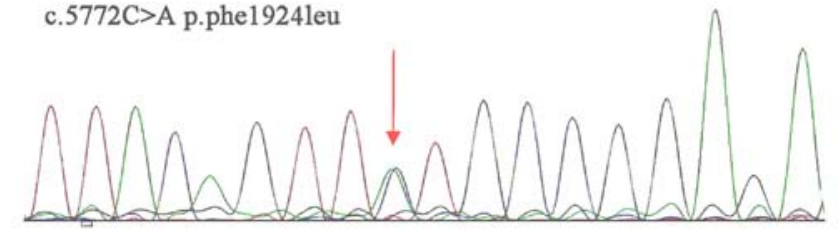

Figure 3. Polycystic kidney and hepatic disease 1 mutations identified in the family with autosomal recessive polycystic kidney disease. Two novel compound heterozygous mutations, (A) c.2876C $>\mathrm{T}$ (p.S959F) and (B) c. $5772 \mathrm{C}>\mathrm{A}$ (p.F1924L), were identified. Mutations are indicated by a red arrow.

prenatal diagnosis of ARPKD using ultrasound alone is not reliable (8). For $35 \%$ of patients with only medullary abnormalities, the standard ultrasound exhibits a normal signal (9). The family in the present study underwent repeated antenatal ultrasound examinations and fetal diagnosis of ARPKD was confirmed at week 23.

Considering the phenotypic variability (1), clinicians should consider the risk of predicting the clinical outcome of a child whose parents had already suffered abnormal pregnancies. The use of $P K H D 1$ sequencing data for clinical decision-making requires caution, particularly in cases where only novel or rare missense variants are identified. In the present study, ultrasound results of the first pregnancy of the couple (for the proband, III:1) exhibited separation of the left renal sinus, enhancement of the right renal parenchymal echo, more echo-free zones in the right kidney, which indicated a number of cystic renal dysplasia, and the right cleft lip and palate.

Multiple lines of evidence support that $P K H D 1$ gene mutations serve a pathogenic role in ARPKD $(1,10)$. All of the identified mutations in $P K H D I$ are distributed throughout the gene without evidence of clustering at specific sites. The majority of these mutations are compound heterozygous mutations, which consist of 332 missenses (252 possibly/probably pathogenic and 80 single nucleotide polymorphisms), 55 nonsense, 86 frame shift and 58 silent mutations (1,9,11-13). The two mutations identified in the present study, to the best of our knowledge, have not previously been reported. Patients with two truncation mutations exhibit a grievous phenotype resulting in perinatal or neonatal demise, whereas patients escaping from neonatal lethality usually possess no more than one amino acid substitution (14). The proband (with two mutations) in the present study died, resulting in a miscarriage, whereas the parents, with only one of the two gene mutations, had no phenotype. It is hypothesized that the expression of only a minimal amount of functional protein is required for survival during the neonatal period in ARPKD (7). Since there are 67 exons in the PKHDl gene, it is not efficient to screen mutations for this gene by Sanger sequencing. Therefore, targeted NGS which may be of specific interest to identify mutations (2), was employed to identify causative mutations in PKHD1. In the present study, two newly identified mutations (c.2876C $>\mathrm{T}$ and c.5772C $>\mathrm{A}$ ), which caused moderate alterations in the genotype and no changes in the phenotype of the parents, were identified. These observations were consistent with previous reports that patients who survive the neonatal period usually have no more than one missense mutation (8-10).

In conclusion, the present study emphasized the importance of prenatal diagnosis of renal abnormalities by routine fetal anatomic ultrasound and genetic testing for ARPKD. Furthermore, the present study provided details of the changes in fetal biometry over the prenatal and perinatal course of ARPKD with two novel PKHDI mutations.

\section{Acknowledgements}

Not applicable. 


\section{Funding}

The present study was supported by grants from the National Natural Science Foundation of China (grant no. 81300618 to XZ), the Sichuan Provincial Planning Commission Fund (grant no. 17PJ058 to XZ) and the Sichuan Provincial People's Hospital Research Foundation (grant no. 2016LY07 to XZ).

\section{Availability of data and materials}

The datasets used and/or analyzed during the present study are available from the corresponding author on reasonable request.

\section{Authors' contributions}

JW, DQ, JY, DZ, QW and XZ designed the study, conducted the experiments and analyzed the data. JW and $\mathrm{XZ}$ wrote the paper. XJ and XZ recruited the participants and collected clinical information. All of the authors contributed to writing of the paper and approved the final version.

\section{Ethics approval and consent to participate}

The present study was approved by the Medical Ethics Committee of the Sichuan Academy of Medical Sciences \& Sichuan Provincial People's Hospital [no. 2013 Natural Science (04)]. The patients provided written informed consent for participation in the study at Sichuan Provincial People's Hospital.

\section{Patient consent for publication}

The patients received all information regarding this study. Written informed consent for publication in Molecular Medicine Reports was obtained from the patients.

\section{Competing interests}

The authors declare that they have no competing interests.

\section{References}

1. Onuchic LF, Furu L, Nagasawa Y, Hou X, Eggermann T, Ren Z, Bergmann C, Senderek J, Esquivel E, Zeltner R, et al: PKHD1, the polycystic kidney and hepatic disease 1 gene, encodes a novel large protein containing multiple immunoglobulin-like plexin-transcription-factor domains and parallel beta-helix 1 repeats. Am J Hum Genet 70: 1305-1317, 2002.

2. Tavira B, Gomez J, Malaga S, Santos F, Fernandez-Aracama J, Alonso B, Iglesias S, Benavides A, Hernando I, Plasencia A, et al: A labor and cost effective next generation sequencing of PKHD1 in autosomal recessive polycystic kidney disease patients. Gene 561: 165-169, 2015 .
3. Dorn L, Menezes LF, Mikuz G, Otto HF, Onuchic LF and Sergi C: Immunohistochemical detection of polyductin and co-localization with liver progenitor cell markers during normal and abnormal development of the intrahepatic biliary system and in adult hepatobiliary carcinomas. J Cell Mol Med 13: 1279-1290, 2009.

4. Ward CJ, Hogan MC, Rossetti S, Walker D, Sneddon T, Wang X, Kubly V, Cunningham JM, Bacallao R, Ishibashi M, et al: The gene mutated in autosomal recessive polycystic kidney disease encodes a large, receptor-like protein. Nat Genet 30: 259-269, 2002.

5. Pang M, Liu Y, Hou X, Yang J, He X, Hou N, Liu P, Liang L, Fu J, Wang K, et al: A novel APC mutation identified in a large Chinese family with familial adenomatous polyposis and a brief literature review. Mol Med Rep 18: 1423-1432, 2018.

6. Gunay-Aygun M, Font-Montgomery E, Lukose L, Tuchman Gerstein M, Piwnica-Worms K, Choyke P, Daryanani KT, Turkbey B, Fischer R, Bernardini I, et al: Characteristics of congenital hepatic fibrosis in a large cohort of patients with autosomal recessive polycystic kidney disease. Gastroenterology 144: 112-121.e2, 2013.

7. Frank V, Zerres K and Bergmann C: Transcriptional complexity in autosomal recessive polycystic kidney disease. Clin J Am Soc Nephrol 9: 1729-1736, 2014.

8. Xu Y, Xiao B, Jiang WT, Wang L, Gen HQ, Chen YW, Sun Y and Ji X: A novel mutation identified in PKHD1 by targeted exome sequencing: Guiding prenatal diagnosis for an ARPKD family. Gene 551: 33-38, 2014.

9. Gunay-Aygun M, Font-Montgomery E, Lukose L, Tuchman M, Graf J, Bryant JC, Kleta R, Garcia A, Edwards H, Piwnica-Worms K, et al: Correlation of kidney function, volume and imaging findings, and PKHD1 mutations in 73 patients with autosomal recessive polycystic kidney disease. Clin J Am Soc Nephrol 5: 972-984, 2010.

10. Bitarafan F and Garshasbi M: Molecular genetic analysis of PKHD1 mutations in pedigrees with autosomal recessive polycystic kidney disease. Iran J Kidney Dis 12: 350-358, 2018.

11. Gunay-Aygun M, Tuchman M, Font-Montgomery E, Lukose L, Edwards H, Garcia A, Ausavarat S, Ziegler SG, Piwnica-Worms K, Bryant J, et al: PKHD1 sequence variations in 78 children and adults with autosomal recessive polycystic kidney disease and congenital hepatic fibrosis. Mol Genet Metab 99: 160-173, 2010.

12. Datta R, Shah GN, Rubbelke TS, Waheed A, Rauchman M, Goodman AG, Katze MG and Sly WS: Progressive renal injury from transgenic expression of human carbonic anhydrase IV folding mutants is enhanced by deficiency of p58IPK. Proc Natl Acad Sci USA 107: 6448-6452, 2010.

13. O'Meara CC, Hoffman M, Sweeney WE, Tsaih SW, Xiao B, Jacob HJ, Avner ED and Moreno C: Role of genetic modifiers in an orthologous rat model of ARPKD. Physiol Genomics 44: 741-753, 2012.

14. Koga T, Duan H, Urabe K and Furue M: IFN-gamma-positive immunostaining in psoriatic lesional keratinocytes-reply to the comments of McKenzie et al. Eur J Dermatol 13: 99, 2003.

This work is licensed under a Creative Commons Attribution-NonCommercial-NoDerivatives 4.0 International (CC BY-NC-ND 4.0) License. 